Teknokultura

ISSN: $1549-2230$

\title{
El camino de una publicación tecnocientífica: Sociología y tecnociencia
}

\author{
Jesús Alberto Valero-Matas ${ }^{1}$, Juan Romay $\mathrm{Coca}^{2}$
}

Recibido: 4 de noviembre de 2016 / Aceptado: 16 de marzo de 2017

Resumen. El presente artículo aborda el análisis de la trayectoria de la revista Sociología y Tecnociencia que los autores pusieron en escena en enero del año 2010. Realiza un relato de los diferentes aspectos que han caracterizado estos años de andadura de dicha revista, poniendo de relieve las dificultades y alegrías por los que ha ido atravesando la publicación, así como las personas que han colaborado y ayudado a que dicha revista tenga un lugar en el campo de la ciencia y tecnología sociológica.

Palabras clave: sociología, tecnociencia, revista académica.

\section{[en] The path of a techno-scientific journal: Sociology and Technoscience}

\begin{abstract}
This paper addresses the historical analysis of the journal Sociology and Technoscience that the authors put on the scene in January of 2010. It focus on the different aspects that have characterized the journal in these years of histor, highlighting the difficulties and joys that have been going through the publication, as well as the people who have collaborated and helped that the journal has a place in the field of sociological science and technology.
\end{abstract}

Keywords: Sociology, tecnoscience, academic journals.

Cómo citar: Valero-Matas, J.; Romay Coca, J. (2017) El camino de una publicación tecnocientífica: Sociología y tecnociencia, en Teknokultura 14, 171-175.

1 Universidad de Valladolid

E-mail: aleroma@soc.uva.es

2 Universidad de Valladolid

Email: juancoca@soc.uva.es 
Luhmann en su obra Sistemas sociales (1998) habla de la existencia de una serie de sistemas cerrados operacionalmente y con capacidad autopoiética. Este sociólogo habla de la existencia de cuatro grandes sistemas: el social, el psíquico, el natural y el técnico. Dentro de ese gran sistema tetralógico nos vamos a ir encontrando con otros sistemas de menor tamaño. A nuestro juicio, el sistema tecnocientífico es uno de ellos y con el objetivo último de ir conociendo sus entresijos se puso en marcha en el 2010 una publicación que tendría como ámbito de especialización este pequeño sistema.

La revista Sociología y tecnociencia nace, fundamentalmente, como fruto de una intuición y una inquietud de dos profesores del Departamento de Sociología y Trabajo Social de la Universidad de Valladolid, en colaboración con personas preocupadas por recuperar las investigaciones en sociología del sistema tecnocientífico. Cuando comenzamos a publicar la revista resultaba muy evidente que los estudios sociales de la ciencia y la tecnología, provenientes de la filosofía, habían ocupado un terreno fértil y propio de la sociología. De hecho, en 2010 no había ninguna publicación -en el mundo de habla hispana- que tuviese como centro de su interés la sociología del sistema tecnocientífico.

Esta motivación fundamental fue la causa fundamental de intentar promover una publicación de esta índole y, evidentemente, rellenar ese hueco. Ahora bien, como bien expone Luhmann (1983) el análisis de los medios para obtener un fin implica aclarar qué renuncias reclama el desarrollo y la realización de tal fin. Veamos, entonces, cuáles han sido dichas renuncias.

Dado el contexto en el que surge la publicación que nos ocupa, una de las primeras renuncias que se tuvieron que asumir provino de la no aceptación de la exclusividad disciplinaria. Dicho de otra manera, no era conveniente (ni lo sigue siendo) entrar en una especie de guerra absurda con otros campos del conocimiento. La razón fundamental es el convencimiento de que sólo a través de un diálogo interdisciplinar podemos asentar las bases de un conocimiento útil a la sociedad. Pero también existía otra razón para dicha renuncia.

Los responsables últimos de la publicación, el editor y el director, mantenían (y todavía lo mantienen) una estrecha relación con personas del mundo de la filosofía, de la historia, de la pedagogía, de las ciencias de la comunicación o de las didácticas específicas, por poner un ejemplo. Esto hace que la dificultad en la gestión de la revista sea más complicada, lo que implica una renuncia a la facilidad de desarrollo interno de la misma.

Por otro lado, Sociología y tecnociencia nace con el convencimiento de que la ciencia y la tecnología están afectando a numerosos ámbitos del sistema social. Esto supone que la importancia de disciplinas como la sociología del sistema tecnocientífico sea de enorme relevancia en la actualidad. Esta convicción nos obliga a renunciar a análisis más amplios y diversos de la realidad social, aunque no menos fructíferos.

Uniendo todas estas perspectivas se decidió que Sociología y tecnociencia fuese una publicación inclusiva, abierta, plural, a-ideológica y con la intención de que atendiese a todas las derivas de investigación que se han ido desarrollando en los últimos años en la sociología del conocimiento o en la sociología de la ciencia y la tecnología. Sociología y tecnociencia se gestó, entonces, como un proyecto sociológico abierto a todos los ámbitos del saber, pero con el objetivo de mejorar el aná- 
lisis del propio sistema tecnocientífico y de su impacto social. De ahí que los trabajos en el ámbito de la teoría social (bien sociológicos, bien filosóficos) hayan tenido un lugar destacado en la publicación. A su vez, también han tenido un lugar destacado los trabajos sobre comunicación, los filosófico-teóricos y los psicológicos.

Esta visión amplia se intenta mostrar en el título de la publicación al hacer mención a la tecnociencia. Ahora bien, tecnociencia entendida como sistema social, no como un ámbito disciplinar posmoderno. Esto no quiere decir que el planteamiento de la revista sea moderno o posmoderno. Como acabamos de decir esta publicación es plural, por ello se desea que en sus páginas se puedan ver estudios sobre la biotecnología, la política, la economía, el derecho, la educación, etc. desde una perspectiva sociológica (directa o indirectamente). Así mismo, también tienen cabida la axiología, la teología, etc. Por lo tanto, pese a que es una revista de ciencias sociales también está abierta a las ciencias formales, a las ciencias experimentales y a las humanas.

La revista nació con gran ilusión y, desde su gestación, siempre hemos tenido pretensiones de fuese una revista de calidad y de referencia. Además, antes de que ésta viese la luz éramos conscientes de que el acceso abierto es necesario, incluso fundamental, en nuestra realidad actual. Por ello hemos optado por una revista abierta y sin costes para los autores. A día de hoy Sociología y tecnociencia se encuentra bajo la siguiente licencia: Creative Commons Reconocimiento CompartirIgual 4.0 Internacional License.

Además, y pese a que algunas bases de datos plantean problemas con ello, optamos por unos números pequeños, muy limitados, con pocos artículos pero de la mayor calidad posible. La idea que tenemos es la de no aburrir al lector con multitud de trabajos que puedan llegar a dispersar. Por ello nuestro objetivo es rondar los cinco trabajos en cada número. Además, y debido al ámbito de especialización de la revista no era lógico pensar que se recibirían multitud de trabajos.

En los inicios de nuestra andadura, el equipo de redacción de Sociología y tecnociencia comprendió que teníamos grave limitaciones, fruto de cierta carencia en la llegada de textos. Por esto hemos tenido que realizar un trabajo muy fuerte en la internacionalización de la revista y en su difusión. Ello ha hecho que cada vez recibamos trabajos de mayor relevancia e interés para nuestra disciplina de conocimiento. Además, y fruto de la exigencia de calidad requerida en los textos el índice de rechazo, sin que esto suponga ningún indicio de calidad, se encuentra entre un 30 y $50 \%$ en función de los años.

Así mismo, una de las primeras apuestas fue la inclusión de la revista en todas las bases de datos posibles. Las primeras respuestas recibidas eran ilusionantes. Comprobar que los artículos comenzaban a moverse a nivel internacional resultaba francamente bonito. Pero dada las exigencias de publicación de las agencias de calidad, de la competencia con otras publicaciones más antiguas y de los rankings universitarios, los comienzos supusieron un enorme trabajo para los responsables de la revista.

Una vez que tuvimos el aval de una buena cantidad de bases de datos, comenzamos a promover la revista en diversos foros académicos, seminarios de investigación, congresos, etc. Incluso llegamos a proponerle al grupo de Sociología del conocimiento y de la ciencia y la tecnología de Federación Española de Sociología 
que la revista se convirtiese en su órgano de expresión, sin perder el carácter de nuestra publicación. Desconocemos la razón, pero por desgracia esta idea nunca se llegó a materializar.

Como el lector habrá podido entrever, la pretensión de esta publicación es llegar a convertirse en la referencia internacional, especialmente en lengua castellana y portuguesa y en el ámbito de especialización de Sociología y tecnociencia. Precisamente esta especialización ha logrado que recientemente se nos incorporase en ESCI (WOS). Para nosotros este hecho es un espaldarazo a todo el esfuerzo realizado y las numerosas horas invertidas en su desarrollo.

A partir de la incorporación de nuestra publicación en Web of Science (WOS) optamos por realizar una fuerte remodelación del equipo de la revista. Por ello, al tiempo que fue remodelado el consejo de redacción, también se produzco un sustancial incremento del consejo asesor nacional e internacional con una mayor presencia de mujeres. Ello fue debido, en buena medida, a la colaboración con la profesora $D^{\mathrm{a}}$ Eulalia Pérez Sedeño y a las subdirectoras de la revista, $\mathrm{D}^{\mathrm{a}}$ Francesca Randazzo y $\mathrm{D}^{\mathrm{a}}$ Ana M. González. Con ello logramos subsanar un problema de equidad interna que arrastrábamos desde los inicios de la publicación.

Sociología y tecnociencia tiene una línea editorial amplia, teniendo cabida textos cualitativos y cuantitativos. Ahora bien, cuando uno revisa lo que se ha ido publicando en estos años de vida de la revista se ve claramente que los trabajos de corte cualitativo y de comprensión de la realidad del sistema tecnocientíficos han tenido una fuerte impronta en los diferentes números.

El impacto de esta publicación, si nos guiamos por el índice de hirsch (índice h) se encuentra entre el 5 y el 6. Por otro lado, y actualmente, su índice g es de 7. Así mismo, según la base de datos MIAR su índice compuesto de difusión secundaria (ICDS) es de 9,3.

Actualmente el equipo que dirige el camino de la revista Sociología y tecnociencia tiene dos líneas de trabajo principales. La primera consiste en seguir internacionalizando la revista, incrementando la difusión y su impacto académico. La segunda, y relacionada con la anterior, consiste en lograr que firmas de reconocido prestigio internacional publiquen en nuestra revista. Por ello hemos creado la sección firma invitada con el objetivo de hacer públicos trabajos de personas de alto nivel y fidelizar así los lectores. En este sentido es obligado destacar la presencia de un trabajo del profesor Dimitri Ginev (2017), uno de los máximos exponentes de la epistemología social de la ciencia y del conocimiento.

El futuro de la revista que aquí nos ocupa se podrá seguir consolidando a través del mantenimiento de la especialización y del cuidado de la calidad en la publicación de artículos. Estos aspectos no pueden estar regidos única y exclusivamente por elemento bibliométricos. La calidad de un trabajo o de una revista va más allá de los índices de citación y de impacto (Valero Matas et al. 2013).

Sociología y tecnociencia es la primera publicación de este ámbito de conocimiento en Iberoamérica y los responsables de ella desean que sea una de las primeras del mundo. En este sentido, y dado que la revista está ahora en Web of Science, su equipo seguirá trabajando para consolidar su presencia e impacto internacional. Ello no quiere decir que esta publicación científica se olvide del contexto donde nació la publicación pero el imperativo internacional es un condicionante muy fuerte. 


\section{Agradecimientos}

El equipo responsable de la revista agradece a todas y cada una de las personas que hacen que este ilusionante proyecto sea posible: autores, lectores, asesores, etc. A todos ellos gracias infinitas.

\section{Referencias bibliográficas}

Luhmann, N. (1983): Fin y racionalidad en los sistemas, Madrid: Editora Nacional.

Luhmann, N. (1998): Sistemas sociales, Barcelona: Anthropos.

Ginev, D. (2017). Interpretive Internalism In The Time Of Technoscience. Sociología y tecnociencia/Sociology \& Technoscience/Sociologia e tecnociência, 2(6), 01-16.

Valero Matas, J. A.; Jiménez García, E. y Coca, J. R. (2013): "La perspectiva sociohermenéutica en la evaluación del trabajo científico en España”, Investigación bibliotecológica, Vol. 27, N 59, pp. 15-34. 